Revista de Psicología de la PUCP. Vol. XIX, 2, 2001

\title{
Predictores conductuales y sociodemográficos de riesgo para el contagio del VIH y otras ITSs
}

\author{
Julio Piña ${ }^{1}$, Luis Fierros ${ }^{2}$ y Humberto de la Cruz Moreno ${ }^{3}$ \\ Universidad de Sonora
}

\begin{abstract}
Se evaluó a 1435 participantes con una prueba de laboratorio para detectar anticuerpos al VIH u otras infecciones de transmisión sexual (ITSs) entre 1999 y el 2000. Los resultados mostraron que (a) la mayoría acudió al Centro de Información para la Salud por primera vez; (b) La prueba de Elisa fue la más frecuentemente usada; (c) la mayoría de participantes son del género femenino y (d) el rango de edad está entre los 15 y 44 años. En relación con las conductas de riesgo, las del tipo sexual fueron las de mayor frecuencia; entre las adictivas, las del consumo de droga por vía intravenosa y alcohol fueron las más frecuentes. Correlaciones significativas, confirmadas con la prueba de bondad de ajuste Kolmogrov-Smirnov, fueron halladas entre las variables sociodemográficas. Al analizar la dimensión sexual sola y con droga intravenosa hubo una correlación significativa de la variable conductas de riesgo frente al resto de variables.

Palabras clave: VIH, Infecciones de Transmisión Sexual, variables sociodemográficas, conductas de riesgo y conductas adictivas.
\end{abstract}

\section{Behavioral Risk and Sociodemographic Predictors related to HIV infection and other STI's}

One thousand thirty-five participants were tested in laboratory in order to detect HIV or other sexually transmitted infection (STI) antibodies between 1999 and 2000 . The results showed that: (a) most of them attended for the first time the Health Information Center; (b) the Elisa test was the most frequently used; (c) the majority were women, and (d) their age range was from 15 to 44 years old. With regard to risk behaviors, the sexual ones were the most frequent; in relation to addictive behaviors, drug consumption by intravenous way and alcohol use were the most frequent ones. Significant correlation, confirmed by Kolmogrov-Smirnov test, were found among the sociodemographic variables. When analyzing sexual behavior alone and with alcohol consumption, a significant correlation was found in risk behaviors compared to the other variables.

Keywords: HIV, Sexually Transmitted Infection, sociodemographic variables, risk behavior and addictive behavior.

1 Coordinador de Investigación en el Programa Estatal para la Prevención y Control del VIH/SIDA y otras ITSs. (Secretaría de Salud del estado de Sonora). Licenciado en psicología por la Universidad del Noroeste (Hermosillo, Sonora). Es actualmente estudiante de la maestría en psicología de la U. Sonora.jpina@kunkaak.psicom.uson.mx.

2 Profesor-Investigador de la Maestría en Psicología. Magíster en modificación de conducta y Licenciado en psicología (UNAM). Investiga actualmente problemas de comportamiento y conductas de riesgo en adolescentes. Correo electrónico: Ifierros@kunkaak.psicom.uson.mx. 

En los Estados Unidos de Norteamérica se cuenta con dos fuentes de evaluación que tienen por objeto recoger información sobre la práctica de conductas de riesgo de infección por el VIH, en la población en general: el Sistema de Datos sobre Consejería y Pruebas de Detección de anticuerpos al VIH, por un lado, y la Encuesta Nacional sobre Salud, por el otro (Anderson, 1996). La primera de esas fuentes, de acuerdo con este autor, tiene la ventaja de proveer información valiosa para planificar y evaluar el impacto que los programas de consejería y realización voluntaria de pruebas de laboratorio tienen sobre el posible cambio de conductas de riesgo por conductas preventivas.

Dicho sistema que se fundamenta en la interacción de la díada consejería-prueba, se ha llegado incluso a considerar como un modelo apropiado para educar en torno al VIH/SIDA, a las personas que se realizan la prueba de detección de anticuerpos al VIH (Centers for Disease Control and Prevention, 2000), o bien como una estrategia efectiva para reducir la práctica de conductas de riesgo asociados a la citada enfermedad (Kamenga, Sweat, De Zoysa, Dallabetta, Coates $e t$ $a l ., 2000)$. El problema que se observa, a propósito de ese sistema, es que pareciera no existir consenso alguno acerca de, primero, qué medir o evaluar respecto a la variable conductas de riesgo, o de otros eventos de naturaleza psicológica y social; y segundo, sobre la definición, criterios y estrategias de cambio conductual al adoptar a la consejería como su programa base.

En efecto, mientras en algunos casos su empleo se reduce a medir aspectos relacionados a la percepción de riesgo que se tiene sobre el potencial contagio del VIH y un tipo específico de conductas de riesgo

3 Coordinador General del Programa Estatal para la Prevención y Control del VIH/SIDA y otras ITSs. (Secretaría de Salud del estado de Sonora). Es Licenciado en Medicina por la UNAM y el Instituto Nacional de Salud Pública. Investiga el control de infecciones de transmisión sexual en la población. Correo electrónico: hcm57@prodigy.net.mx. 
en hombres con preferencias homo o bisexuales (Wardropper, Sankar y Ong, 1995), en otros se trata de establecer, en mujeres jóvenes y adultas, qué conductas de riesgo - por ejemplo, el número de parejas sexuales y uso de preservativo en el último año- pueden constituirse como predictores importantes para que personas de ese grupo social se realicen la prueba de detección de anticuerpos (Miller, Hennessy, Wendell, Webber y Schoenbaum, 1996); o bien se analiza cómo es que los conocimientos, actitudes y conductas de riesgo se relacionan cuando adolescentes y jóvenes entre los 13 y 23 años se realizan la prueba en cuestión (Rotheram-Borus, Gillis, Reid, Fernandez y Gwadz, 1997).

Asimismo, hay aquellos en los que se intenta obtener información puntual en lo tocante a los motivos o razones de por qué las personas se realizan las pruebas, como cuando se busca confirmar el estatus de seropositividad, a fin de iniciar una nueva relación de pareja (Coyle, Knapp y O' Dea, 1996); u otros que pretenden afirmarse como programas de amplio espectro, orientados de manera particular a promover el cambio de conductas de riesgo por conductas preventivas.

Dentro de estos últimos se encuentra un estudio realizado por Wenger, Greenberg, Hilborne, Kusseling, Mangotich y Shapiro (1992). Se contempló una muestra de 2,196 estudiantes universitarios, todos con preferencias heterosexuales, y en el que se encontró que de una submuestra conformada por 435 sujetos, que fueron asignados a uno de tres grupos: control, educación y educación con prueba. Los de este último reportaron en una evaluación de seguimiento a los seis meses, haber mejorado sustancialmente su comunicación frente a sus parejas (preguntándoles sobre su estatus de seropositividad respecto del VIH/SIDA), aunque se observó que entre los tres grupos no se manifestaron diferencias importantes en el número de parejas sexuales y en lo tocante al uso consistente del preservativo.

También se encuentra el conducido por Ickovics, Morrill, Beren, Walsh y Rodin (1994), en el que se contó con un grupo de 230 mujeres, de las cuales 152 formaron parte del grupo consejería con prueba 
Predictores conductuales y sociodemográficos de riesgo para el contagio del VIH...

y 78 del grupo control (o de comparación); los autores destacan que el promedio de riesgo sexual fue más bajo en el primero de los grupos, que adicionalmente mencionaron tener una menor cantidad de pensamientos "intrusivos" sobre SIDA, así como una estimación más real sobre la probabilidad de contagio por el VIH; no obstante en una evaluación de seguimiento a los tres meses, no se reportó diferencia entre ambos grupos respecto al ejercicio instrumental de conductas de riesgo ( $v g$. diferentes parejas sexuales y uso de preservativo).

Asimismo, Kamb, Fishbein, Douglas, Rhodes, Rogers et al. (1998), en un estudio que se llevó al cabo entre 1993 y 1996 en diferentes ciudades de los Estados Unidos de Norteamérica, de un total de 5,578 participantes, a los cuales se les había notificado resultados negativos a la prueba de detección de anticuerpos al VIH y que fueron asignados a 4 grupos de intervención para probar la eficacia de la consejería para prevenir la infección por VIH u otras ITSs - consejería mejorada, consejería breve, mensajes didácticos (los tres con periodos de seguimiento a los 3, 6, 9 y 12 meses), así como el de mensajes didácticos sin evaluación de seguimiento programada-, se observó que los mejores beneficios se reportaron en los dos primeros grupos, tanto en lo que se refiere al uso del preservativo como en una reducción significativa de ITSs a lo largo de los diferentes periodos de seguimiento.

De igual manera el estudio de Leaity, Sherr, Wells, Evans, Miller, Johnson y Elford (2000), quienes trabajaron con una muestra de 1,446 sujetos, de los cuales $721(50.6 \%)$ se repitieron la prueba de detección de anticuerpos al VIH. Al margen de la preferencia sexual de los participantes (homosexuales, y hombres y mujeres heterosexuales), no se reportaron diferencias significativas en la práctica de sexo no protegido entre la realización de la primera y segunda pruebas; si bien se hace notar que particularmente $50 \%$, tanto de los hombres como de las mujeres heterosexuales, concibieron al conocimiento de los resultados de las pruebas como una medida importante para reducir la práctica de conductas de riesgo. 
Con objeto de obtener información sobre el tipo de variables, y cómo es que interactúan entre ellas en un grupo de personas que acuden al Centro de Información para la Salud para realizarse alguna de las pruebas de laboratorio que tienen que ver con el problema del VIH/SIDA y otras ITSs, se planteó la presente investigación cuyos objetivos fueron los siguientes:

- Explorar predictores significativos de ciertas variables conductuales y sociodemográficas en personas que se realizan voluntariamente la prueba de detección de anticuerpos al VIH u otras ITSs.

- Analizar la posible correlación de la variable conductas de riesgo (desagregada por dimensión) respecto de las variables sociodemográficas, preferencia sexual y conductas adictivas.

- Analizar la posible correlación de las variables edad (por subgrupo) respecto de determinadas variables sociodemográficas, conductas de riesgo, preferencia sexual y conductas adictivas.

\section{Metodología}

\section{Participantes}

En el estudio participaron 1,435 personas, de las cuales el $40.5 \%$ eran hombres y el $58.5 \%$, mujeres; en 15 casos $(1 \%)$ no se obtuvo la información sobre el sexo. La mayoría acudió de manera voluntaria al Centro de Información para la Salud (CISAL) a solicitar se les practicaran las pruebas de laboratorio para identificar anticuerpos al VIH o de otras infecciones de transmisión sexual. Las edades oscilaron en un rango de 11 a 75 años, con una media de 28.7 años y una desviación estándar de 10.01 .

\section{Instrumentos}

Se utilizó un formato de entrevista estructurada que consta de un conjunto de reactivos subdivididos en las siguientes áreas: (a) fecha 
Predictores conductuales y sociodemográficos de riesgo para el contagio del VIH...

del contacto, tipo de contacto (primera vez o seguimiento), y tipo de estudio que se solicita o por el cual se remite a la institución (detección de anticuerpos al VIH, pruebas confirmatorias de seropositividad al VIH, detección de ITSs, etcétera); (b) variables sociodemográficas que incluyen sexo, edad, estado civil, escolaridad y ocupación, y (c) variables asociadas a conductas y preferencia sexual: ejercicio instrumental de conductas de riesgo - por la vía sexual, fundamentalmente-, preferencia sexual y conductas adictivas - consumo de drogas por las vías oral e intravenosa, así como consumo de alcohol-.

Las variables que son investigadas son entendidas del siguiente modo: Conductas de riesgo son aquellas que hacen referencia al hecho de practicar cualquier modalidad de intercambio sexual con penetración y sin el uso consistente de preservativo; preferencia sexual hace referencia a la libre elección de pareja con la que se mantiene intercambio sexual con penetración, sea homosexual, heterosexual o bisexual; conductas adictivas se entienden como el consumo de cualquier droga (sustancia psicoactiva), sea esta inyectada o consumida por vía oral.

\section{Procedimiento}

En el presente estudio de corte transversal (en sus modalidades descriptivo y correlacional), todos los sujetos fueron expuestos a las siguientes condiciones. Primera, inicialmente eran atendidos por personal del área de psicología o promoción de la salud, quienes posterior al establecimiento del rapport y a la confirmación de los motivos de su visita, les solicitaban respondieran de forma voluntaria a una serie de preguntas contenidas en el formato de entrevista estructurada; segunda, a todos los sujetos se les brindaba una sesión de asesoría/consejería - cuya duración en promedio fue de $25 \mathrm{minu-}$ tos- en la que se les ofrecía información general relacionada a los aspectos preventivos del VIH/SIDA y otras ITSs; tercera, se les entregaba un pase especial para que acudieran al Centro Estatal de la Transfusión Sanguínea a fin de realizarse la prueba correspondiente; 
$y$, finalmente, pasados entre 10 a 20 días, retornaban a recoger los resultados, los cuales invariablemente les eran entregados de manera individual, pudiendo en ocasiones, especialmente cuando así se solicitaba, ampliar la información proporcionada en la primera sesión de asesoría/consejería.

\section{Análisis estadístico}

Del total de las variables se extrajeron las frecuencias y porcentajes correspondientes. Posteriormente con el paquete estadístico SPSS para Windows, versión 8.0, se obtuvieron diferentes correlaciones entre las variables de interés, empleando para dicho propósito el estadístico rho de Spearman, aceptándose niveles de significancia de $p<.01$ y $p<.05$.

Un segundo paso consistió en efectuar un análisis no paramétrico para extraer la bondad de ajuste de los datos relacionados con la variable riesgo, para lo cual se empleó la prueba de KolmogorovSmirnov, a fin de identificar el grado de acuerdo entre la distribución de un conjunto de valores muestreados (valores observados) y alguna distribución teórica específica, que se representa como lo que podría esperarse según la hipótesis nula. Para este análisis se consideró a la variable conductas de riesgo y sus respectivas dimensiones, en cuanto a las variables edad, sexo, preferencia sexual y conductas adictivas.

Finalmente, se llevó a cabo la elaboración de una tabla de contingencia (CROSSTAB, por sus siglas en inglés). El objeto era mostrar la forma en que se distribuyen los casos de dos o más variables que tienen un número ilimitado de diferentes valores para calcular las medidas que cuantifican la magnitud de la relación observada entre la variable edad con respecto de las variables: sexo, estado civil, escolaridad, conductas de riesgo, preferencia sexual y conductas adictivas. Para dicho 
Predictores conductuales y sociodemográficos de riesgo para el contagio del VIH...

cálculo se recurrió a dos de las medidas de asociación contempladas en el paquete estadístico, que son el coeficiente $\mathrm{V}$ de Cramer y el coeficiente de Contingencia, dado que constituyen opciones valiosas que permiten analizar variables que tienen más de dos valores.

\section{Resultados}

En términos generales se encontró que la mayoría de las personas que acudieron al CISAL entre 1999 y el año 2000 eran contactos de primera vez ( $n=1,003 ; 69.9 \%)$, un importante número lo hizo por cuestiones de seguimiento $(n=402 ; 28.6 \%)$; es decir, se trataba de personas que requerían la prueba confirmatoria de anticuerpos al VIH - conocida como Western Blot-, para confirmar casos de ITSs, o bien para monitorear cargas virales o las cuentas de CD4, especialmente de personas que viven con el VIH/SIDA en sus diferentes estadios.

Por otro lado, se realizaron más pruebas del tipo ELISA, como primera prueba de elección para detectar anticuerpos al VIH $(n=671$; $46.8 \%$ ), así como en combinación con otras pruebas, que incluían hepatitis B, hepatitis C y confirmatoria del tipo Western Blot ( $n=145$; $10.2 \%$ ); el resto se distribuye en pruebas independientes de hepatitis $\mathrm{B}$, hepatitis C, VDRL o combinadas de estas, si bien no representan un número o porcentaje significativos.

Asimismo, del total de las 1,435 personas, un elevado porcentaje se concentra en los subgrupos de edad que van de los 15 a 24 y 25 a 44 años $(n=1,249 ; 86.9 \%)$, seguidos de los subgrupos de 45 a 64 años y 0 a $14^{4}$ (Cuadro 1 ).

4 No obstante los autores del presente trabajo consideran que ese sistema de categorización por edades no es el más apropiado, el mismo se utilizó debido a que históricamente en el estado de Sonora se ha empleado uno similar para todo lo relacionado con la morbi-mortalidad del VIH/SIDA. 


\section{Cuadro 1}

Frecuencia y porcentaje de los participantes de acuerdo con la edad

\begin{tabular}{|c|cr|}
\hline Subgrupo de edad & Frecuencia & $\%$ \\
\hline 0 a 14 años & 65 & 4.5 \\
15 a 24 años & 483 & 33.6 \\
25 a 44 años & 766 & 53.3 \\
46 a 64 años & 102 & 7.1 \\
65 o más años & 4 & 5 \\
Se ignora & 15 & 1.0 \\
\hline Totales & $N=1,435$ & 100.0 \\
\hline
\end{tabular}

Adicionalmente, se observa que la mayoría de las personas se mantienen en calidad de solteras/os $(n=966 ; 67.3 \%)$, casadas/os $(n=201 ; 14.0 \%)$ o viven en unión libre $(n=109 ; 7.6 \%)$, con una baja cantidad de divorciadas/os o viudas/os, aunque en esta variable se ignora una importante cantidad de casos $(n=91 ; 6.3 \%)$.

Finalmente, en escolaridad se encontró que $n=369(25.7 \%)$ contaban con estudios parciales o totales de primaria; $n=364(25.4 \%)$ de secundaria; $n=225(15.7 \%)$ de profesional y $n=175(12.2 \%)$ de bachillerato, con pocos casos de personas con estudios técnicos o ningún tipo de estudio, aunque también se ignora una importante cantidad de casos $(n=161 ; 11.2 \%)$.

Un aspecto de suma relevancia en este trabajo lo constituyó el tipo de ejercicio instrumental de conductas de riesgo por el que las personas acuden a realizarse alguna de las pruebas mencionadas. Estas conductas se desglosaron en: sexual para la población en general $(n=841 ; 58.6 \%)$, sexual para personas que se dedican al sexo-servicio $(n=361 ; 25.2 \%)$, sexual con consumo de droga por vía intravenosa $(n=84 ; 5.9 \%)$, consumo de droga por vía intravenosa $(n=14 ; 1 \%)$ y por donación de sangre o padecer hemofilia $(n=11 ; 8 \%)$. En los demás casos de menor cuantía, se trató de personas que acudieron por estar embarazadas; porque se les solicitaban los resultados para efectuar 
Predictores conductuales y sociodemográficos de riesgo para el contagio del VIH...

algún trámite legal; o por ser menores de edad que se encontraban bajo la custodia de instituciones diversas, como el Consejo Tutelar para Menores o la Casa Hogar Jineseki que alberga a los conocidos como "niños de la calle".

En cuanto a la variable preferencia sexual, destaca el hecho de que $n=997(67.3 \%)$ de las personas reportan ser heterosexuales, $n=126(8.8 \%)$ homosexuales y $n=44(3.1 \%)$ bisexuales. En esta variable es necesario tener en cuenta que se desconoce la preferencia sexual de $n=268(18.6 \%)$ personas que son una frecuencia y un porcentaje que pueden limitar un posterior análisis de los resultados; una situación similar se encontró con la variable conductas adictivas de los cuales se desconoce su incidencia en 1,181 casos, es decir, más de tres cuartas partes del total.

A partir de estos resultados y de las limitaciones ya señaladas, se procedió a establecer algunas correlaciones entre las variables de interés, empleando el estadístico rho de Spearman. Los resultados de dichas correlaciones, que se muestran en el Cuadro 2 , sugieren importantes correlaciones, estadísticamente significativas, en casi todas las variables.

\section{Cuadro 2}

Correlaciones obtenidas con rho de Spearman entre variables sociodemograficas y las relacionadas con conductas de riesgo y adictivas, así como a preferencia sexual

\begin{tabular}{|lllllll|}
\hline Variables & 2 & 3 & 4 & 5 & 6 & 7 \\
\hline 1. Edad & $.194^{* *}$ & $.175^{* *}$ & -.038 & -.051 & .030 & $-.193^{* *}$ \\
2. Sexo & & $-.134^{* *}$ & -.098 & -.021 & $-.260^{* *}$ & $-.204^{* *}$ \\
3. Estado civil & & & $.112^{* *}$ & $-.075^{* *}$ & $.174^{* *}$ & .027 \\
4. Escolaridad & & & -.050 & $.221^{* *}$ & -.020 \\
5. Conductas de riesgo & & & & $-.067^{* *}$ & .042 \\
6. Preferencia sexual & & & & .039 & \\
7. Conductas adictivas & & & & & \\
\hline
\end{tabular}

${ }^{* *} p<.01$ 
Los anteriores resultados nos indican que prácticamente las variables de tipo sociodemográficas correlacionan entre sí, mientras que las variables relacionadas a los dos tipos de conductas, riesgo y adictivas, parecen no tener un poder explicativo importante. En efecto, la variable conductas de riesgo, dando por descontadas las correlaciones con el estado civil (que como se recordará la mayoría eran personas solteras/os, casadas/os o vivían en unión libre) y con preferencia sexual (la mayoría, heterosexuales), no demostró tener un peso mayor al esperado; el caso de las conductas adictivas se puede explicar por el hecho de que no se contabilizaron muchos de los datos sobre su práctica en la población estudiada.

\section{Cuadro 3}

Prueba de Kolmogorov-Smirnov con la variable conductas de riesgo en sus diferentes dimensiones

\begin{tabular}{|c|c|c|c|c|}
\hline \multirow[t]{2}{*}{ Conductas de riesgo } & & \multicolumn{3}{|c|}{$\begin{array}{c}\text { Prueba de Kolmogorov-Smirnov } \\
\text { para una muestra }\end{array}$} \\
\hline & Media $^{a}$ & D E $\mathrm{a}^{\mathrm{a}}$ & Valores & Nivel de significación \\
\hline \multicolumn{5}{|l|}{ Sexual } \\
\hline 1. Edad & 2.75 & 1.95 & 10.897 & .001 \\
\hline 2. Sexo & 1.58 & .52 & 11.083 & .001 \\
\hline 3. Preferencia sexual & .99 & .62 & 10.683 & .001 \\
\hline 4. Conductas adictivas & .30 & .74 & 14.325 & .001 \\
\hline \multicolumn{5}{|l|}{ Droga intravenosa } \\
\hline 1. Edad & 2.79 & .89 & 1.424 & .05 \\
\hline 2. Sexo & 1.64 & .50 & 1.521 & .05 \\
\hline 3. Preferencia sexual & .86 & .53 & 1.463 & .05 \\
\hline 4. Conductas adictivas & .43 & .94 & 1.728 & .05 \\
\hline \multicolumn{5}{|l|}{ Sanguinea } \\
\hline 1. Edad & 2.36 & 1.03 & 1.213 & .106 \\
\hline 2. Sexo & 1.45 & .52 & 1.172 & .128 \\
\hline 1. Preferencia sexual & .91 & .30 & 1.750 & .05 \\
\hline 2. Conductas adictivas & .36 & .92 & 1.563 & .05 \\
\hline \multicolumn{5}{|c|}{ Sexual con droga intravenosa } \\
\hline 1. Edad & 2.67 & .68 & 3.206 & .001 \\
\hline 2. Sexo & 1.60 & .52 & 3.575 & .001 \\
\hline 3. Preferencia sexual & .99 & .69 & 3.319 & .001 \\
\hline 4. Conductas adictivas & .61 & 1.13 & 3.947 & .001 \\
\hline
\end{tabular}

"La prueba de distribución es normal y ambas medidas se calcularon de los datos. 
Predictores conductuales y sociodemográficos de riesgo para el contagio del VIH...

Con el propósito de evaluar hasta qué punto la variable conductas de riesgo pudiera confirmar los resultados antes descritos, se realizó una prueba de bondad de ajuste de los datos o puntuaciones observadas y alguna distribución teórica específica. Para ello se utilizó la prueba de Kolmogorov-Smirnov, que se caracteriza por mostrar en dos distribuciones, la esperada y la teórica, la menor o mayor divergencia de los datos (Silva, 1998). En el Cuadro 3 se presentan los resultados, tal como se puede apreciar los valores elevados en la dimensión sexual, así como los obtenidos en las dimensiones droga intravenosa y sexual con droga intravenosa, confirman la significancia estadística de los resultados a $p<.001 \mathrm{y}<.05$.

Estos hallazgos suponen que, si bien la variable conductas de riesgo, especialmente cuando es analizada en su conjunto, no evidenció tener un peso explicativo frente al resto de las variables, sí juega un papel más relevante cuando se le analiza de manera desagregada. En el Cuadro 3 se muestra que las dimensiones sexual y sexual con droga intravenosa, sobre todo la primera, obtuvieron valores elevados y significancia estadística. Esto nos conduce a plantear que es necesario efectuar análisis de este tipo de variable tomando en cuenta a cada una de las dimensiones incluidas en ella, pues de otra suerte se podría obscurecer la relevancia de dicha variable al momento de explicar cómo y en qué forma correlaciona con otras variables.

Por último, con objeto de analizar hasta qué punto la variable edad pudiera también tener un peso explicativo frente al resto de las variables, se procedió a elaborar una tabla de contingencia, considerando dos de las medidas de asociación: la $\mathrm{V}$ de Cramer y el coeficiente de Contingencia; en el Cuadro 4 se presentan los resultados obtenidos.

Se observa que las medidas de asociación, tanto con el coeficiente $\mathrm{V}$ de Cramer como en el de Contingencia, demuestran que existen asociaciones estadísticamente significativas entre la variable edad y otras variables sociodemográficas, no así con la variable conductas 
de riesgo, aunque habría de tenerse en cuenta lo comentado en los resultados que se muestran en el Cuadro 2; empero, por otro lado, hay que hacer notar que sí evidenció, no obstante los valores bajos, asociación significativa con la variable conductas adictivas, que bien pudiera deberse al hecho que quien practica este tipo de conductas se encuentran justamente entre los subgrupos de edad que van de los 15 a 24 y 25 a 44 años.

\section{Cuadro 4}

Tabla de Contingencia que relaciona la variable edad con el resto de las variables de interés, a partir de dos medidas de asociación

\begin{tabular}{|lcc|}
\hline Variables & \multicolumn{2}{c|}{ Medidas de Asociación } \\
& Coeficiente V de Cramer & Coeficiente de Contingencia \\
\hline Edad-Sexo & $.171^{* * *}$ & $.235^{* * *}$ \\
Edad-Estado civil & $.152^{* * *}$ & $.321^{* * *}$ \\
Edad-Escolaridad & $.148^{* * *}$ & $.341^{* * *}$ \\
Edad-Conductas de riesgo & $.080^{*}$ & .220 \\
Edad-Preferencia sexual & $.106^{* *}$ & $.180^{* *}$ \\
Edad-Conductas adictivas & $.187^{* * *}$ & $.351^{* * *}$ \\
\hline $\begin{array}{l}* * p<.01 \\
* * *\end{array}<.001$
\end{tabular}

\section{Discusión}

Si se consideran los resultados obtenidos en este estudio, de entrada nos parece oportuno centrar la discusión de los mismos en dos aspectos. Por un lado, en la importancia que reviste su conocimiento para poder así contar con mejores elementos que nos permitan planear 
Predictores conductuales y sociodemográficos de riesgo para el contagio del VIH...

estrategias de prevención del VIH/SIDA y otras ITSs en el contexto de las actividades de asesoría/consejería; y, por el otro, en el contexto global de su instrumentación en el terreno social, con especial referencia en aquellos subgrupos de edad y personas que practican conductas de riesgo del tipo sexual o sexual con adictivos (droga intravenosa).

En efecto, tal y como se ha señalado por diferentes autores (Beardsell, 1994; Centers for Disease Control and Prevention, 1997; Miller, Simon, Miller, Long, Yu y Asch, 1999; Nyamathi, Stein y Swanson, 2000; Wolistky, Mac Gowan, Higgins y Jorgesen, 1997), si alguna utilidad pudiera tener la realización de las diferentes pruebas de laboratorio que las personas se realizan en relación con los problemas de salud multicitados, y las actividades de asesoría/consejería que se conducen, es justamente la de ofrecer información valiosa y oportuna para diseñar estrategias de intervención orientadas al cambio de conductas de riesgo por conductas preventivas.

Sin embargo, sobre esto último es preciso reconocer algunas limitaciones. Primero, a pesar de que, al menos en el caso de nuestro estudio, a la totalidad de las personas que acuden al CISAL a realizarse alguna de las pruebas ya mencionadas, se les brinda una sesión (y eventualmente dos) de asesoría-consejería sobre el VIH/SIDA y otras ITSs, resulta que no siempre se suele definir con la precisión debida qué es lo que se deberá entender por asesoría o consejería (Bronfman y López, 1997), de tal suerte que a falta de dicha definición y del establecimiento de criterios claros sobre la forma en que operan en la práctica, los objetivos para la prevención del VIH/SIDA pierden en muchas ocasiones la validez requerida, en el sentido de estar en condiciones de poder generalizar los resultados.

Segunda, las propias características de los instrumentos de medida que se utilizan, como el formato de entrevista estructurada aquí reportado, plantea serias dudas sobre su pertinencia o uso adecuado, especialmente cuando se observa que en las variables relacionadas 
con ambos tipos de conductas — sexuales y adictivos-, así como con la variable preferencia sexual, se evidenciaron vacíos importantes. Por ello, suponemos que los hallazgos aquí obtenidos, no obstante hayan demostrado ser estadísticamente significativos, deberán necesariamente tomarse con las precauciones que a merita el caso.

Tercera, suponer que el cambio de conductas de riesgo por preventivas sucederá debido a la influencia directa de una o dos sesiones de asesoría/consejería a la que son expuestas las personas, conlleva un profundo desconocimiento de cómo opera la variable psicológica; cómo y con qué propiedades del ambiente interactúa en ejercicio de sus relaciones funcionales; qué efectos producen sobre el comportamiento de una persona en las distintas situaciones interactivas (directos o indirectos, mediatos o inmediatos, etcétera), entre otras. De hecho, suponiendo que en todas y cada una de las sesiones de asesoría/ consejería a las personas se les ofreciera información completa, útil y pertinente sobre las medidas preventivas - por ejemplo utilizar el preservativo de manera consistente, evitar al máximo posible tener relaciones sexuales con penetración cuando se "sospecha" del estatus de seropositividad de la pareja, por citar dos ejemplos específicos, como se ha señalado en otro lugar (Piña, Márquez y Vera, 1994)-, esa información solo puede tener sentido en la medida en que las personas ya hayan hecho algo práctico antes en relación con dicha información (información como capacidad).

Por ello, resulta extremadamente aventurado asegurar que en el contexto particular de la relación que se da entre dos o más personas -previo o posterior a la realización de alguna prueba de laboratorio- los usuarios de facto adoptarán como suyas las indicaciones o sugerencias sobre la necesidad o importancia de ejercitar instrumentalmente conductas preventivas y, posteriormente, de hacerlo independientemente de la situación interactiva en la que se encuentren. 
Predictores conductuales y sociodemográficos de riesgo para el contagio del VIH...

En última instancia coincidimos en parte con lo señalado por Bronfman y López (1997), cuando se preguntan si la caracterización que se tiene tanto de los servicios de apoyo como de consejería constituye un problema de objeto o de semántica, en el sentido del poco o nulo acuerdo que existe entre los profesionales -o no profesionales- que se encargan de proporcionar cualquiera de ellos a los usuarios. Decimos que en parte, puesto que, si bien es cierto clarificar conceptualmente las acciones que se realizan en torno al VIH/SIDA constituye una tarea fundamental, también lo es el clarificar a qué nos referimos cuando tratamos de la dimensión psicológica de la conducta humana en relación con el problema en cuestión (Bayés, 1992; Piña y Bullé, 1994; Piña, Duarte y Guzmán, 1991).

Téngase presente que la dimensión psicológica de la conducta humana corresponde a la conducta individual (Ribes y López, 1985), y nuestro interés como psicólogos debería centrarse en no sólo conocer o saber que un cierto número de personas ejercitan conductas de riesgo en sus dimensiones sexual o sexual con adictiva, y que por lo regular se ubican en los subgrupos de edad que van de 15 a los 24 y de $\operatorname{los} 25$ a los 44 años, sino también en conocer o saber; es decir, identificar qué variables vinculadas a los estilos interactivos - término tradicionalmente ubicado bajo el rótulo de personalidad- (Ribes y Sánchez, 1990) y qué motivos son los que subyacen al ejercicio de ese tipo de conductas, en cualesquiera situaciones sociales en donde las personas interactúan, variable la segunda que ya ha sido estudiada con estudiantes universitarios y que ha demostrado un poder predictivo importante respecto al ejercicio instrumental de conductas de riesgo o prevención (Piña y Corral, 2001).

Con objeto de superar esas u otras limitantes, como parte de las actividades que se hoy en día se realizan en el Centro de Información para la Salud, y teniendo como sustento un modelo psicológico de salud biológica (Bayés y Ribes, 1992; Ribes, 1990), nos hemos propuesto la tarea siguiente: 
1. Rediseñar los diferentes formatos de entrevista estructurada con los que se cuenta y a partir de los cuales se obtiene información sobre el problema del VIH/SIDA en personas que: (a) asisten por primera vez o (b) en seguimiento, para realizarse alguna de las pruebas de laboratorio.

2. Redefinir y rediseñar las actividades vinculadas a la prevención del VIH/SIDA, tanto en lo que se refiere a los diferentes escenarios institucionales o de otro tipo (sector salud, sector educativo, organizaciones sociales diversas, empresas, etcétera), así como en el contexto de la sociedad en su conjunto. Especial atención, en relación con dichas actividades en el contexto institucional, se ha prestado a la importancia que reviste el necesario establecimiento de criterios claros acerca de lo que se entiende por asesoría/consejería, pues hay que considerar que los mismos se brindan tanto por personal profesional - psicólogos-como por personal habilitado como promotores de salud; así como lo que finalmente se debería entender por prevención y qué estrategias para el cambio conductual podrían resultar más idóneas según los sectores de la población a la que vayan dirigidas las campañas (Piña y Obregón, en prensa).

3. Impulsar un programa de investigación en el que, desde el punto de vista psicológico, nos permita evaluar la pertinencia aplicada del modelo psicológico de salud biológica en los distintos niveles de intervención, que contemple a la prevención de la infección por VIH y otras ITSs, por un lado, así como a la intervención para coadyuvar a mejorar la calidad y tiempo de sobrevida de las personas que viven con VIH/SIDA.

Sobre este último, y en estricto apego a lo señalado en el punto uno y a partir de los hallazgos obtenidos en este trabajo, nos hemos dado a la tarea de sugerir una doble línea de investigación. Una, recurriendo a un diseño longitudinal, en la que se pretende obtener información completa y más confiable de las variables psicológicas y sociales que facilitan o interfieren para que las personas que se realizan las pruebas de detección de anticuerpos al VIH o para otras ITSs 
Predictores conductuales y sociodemográficos de riesgo para el contagio del VIH...

instrumenten conductas de riesgo o prevención, así como para indagar acerca de la real utilidad práctica de las actividades de asesoría/ consejería. Para dicho propósito se ha planteado la necesidad de hacer uso de un nuevo formato de entrevista estructurada que, entre otras cosas, sea lo suficientemente potente para obtener información sobre: antecedentes y conductas de riesgo actuales, tipos de conductas de riesgo y rol que desempeña cada integrante de la pareja (p.e., conducta sexual con penetración entre hombres que tienen sexo con otros hombres, sin uso de preservativo, asumiendo un rol activo o pasivo, etcétera), así como las situaciones sociales -escenarios-en los que dichas conductas se llevan al cabo y los motivos individuales que probabilizan su ocurrencia.

La otra, que se trata propiamente dicho de una investigación de corte experimental, pretende probar la eficacia de un doble programa de intervención preventiva: educación sobre VIH/SIDA y otras ITSs, y entrenamiento en competencias diversas, orientadas a promover el ejercicio instrumental de conductas saludables. Para dicho propósito se ha planteado, tentativamente, un diseño en el que, una vez seleccionadas muestras equivalentes de sujetos por edad, sexo y nivel educativo, se sometan a diferentes condiciones: control; prueba de laboratorio, evaluación conductual y asesoría; prueba de laboratorio, evaluación conductual y plática informal; prueba de laboratorio, evaluación conductual y entrenamiento en competencias conductuales, así como una evaluación de seguimiento para cada grupo.

\section{Referencias}

Anderson, J. E. (1996). CDC data systems collecting behavioral data on HIV counseling and testing. Public Health Report, 111 (Suppl. 1), 129-132.

Bayés, R. (1992). Variaciones sobre un modelo de prevención de enfermedad y su aplicación al caso del SIDA. Acta Comportamentalia, 0, 33-50. 
Bayés, R. y Ribes, E. (1992). Un modelo psicológico de prevención de enfermedad: su aplicación al caso del SIDA. En J. A. Piña (Comp.). Psicología y salud: aportes del análisis de la conducta (pp. 1-21). Hermosillo, México: UNISON.

Bearsdell, S. (1994). Should wider HIV testing be encouraged on the grounds of HIV prevention? AIDS Care, 6, 5-19.

Bronfman, M. y López, M. V. (1997). Los servicios de apoyo para VIH/SIDA: el caso del estado de Morelos. SIDA-ETS, 3, 1-8.

Coyle, A., Knapp, M. y O' Dea, E. (1996). Decision making in HIV testing among a group with low HIV risk. Journal of Evaluation Clinical Practice, 2, 223-230.

Centers for Disease Control and Prevention (1997). HIV couseling and testing in publicy funded sites: 1995 summary report. Atlanta, GA: US Department of Health and Human Services, Centers for Disease Control and Prevention.

Centers for Disease Control and Prevention (2000). National HIV testing day at CDC-funded HIV counseling testing and referral sites at United States, 1994-1998. Morbidity and Mortality Weekly Review, 49, 529-532.

Ickovics, J. R., Morrill, A. C., Beren, S. E., Walsh, U. y Rodin, J. (1994). Limited effects of HIV counseling and testing for women. A prospective study of behavioral and psychological consequences. Journal of the American Medical Association, 272, 443-448.

Kamb, M., Fishbein, M., Douglas, J., Rhodes, F., Rogers, J., Bolan, J., Zenilman, J., Hoxworth, T., Malotte, K., Iatesta, M., Kent, C., Lentz, A., Graziano, S., Byers, R. H. y Peterman, T. S. (1998). Efficacy of risk-reduction counseling to prevent immunodeficiency virus and sexually transmitted diseases. Journal of the American Medical Association, 280, 1161-1167.

Kamenga, M. C., Sweat, M. D., De Zoysa, I., Dallabetta, G., Coates, T. J., Grinstead, O. A., Gregorich, S. E., Heilbron, D. C., Wolf, W. P., Choi, K. H., Schachter, J. Balmer, D. Kihuho, F., Plummer, F., Sangiwa, M. G., Hogan, M., Kilewo, J., Mwacigile, D., Furlonge, C., O’Reilly, K. R., Kalibala, S., Nwowane, B. y van 
Predictores conductuales y sociodemográficos de riesgo para el contagio del VIH...

Praag, E. (2000). The voluntary HIV-1 counseling and testing efficacy study: Designs and methods. AIDS and Behavior, 4, 514.

Leaity, S., Sherr, L., Wells, H., Evans, A., Miller, R., Johnson, M. y Elford, J. (2000). Repeat HIV testing: Hig-risk behaviour or risk reduction strategy? AIDS, 14, 547-552.

Miller, K. S., Hennessy, M., Wendell, D. A., Webber, M. P. y Shoenbaum, E. E. (1996). Behavioral risks for HIV infection associated with HIV-testing decisions. AIDS Education and Prevention, 8, 394-402.

Miller, L. G., Simon, P. A., Miller, M. E., Long, A., Yu, E. I. y Asch, S.M. (1999). High-risk sexual behavior in Los Angeles: Who receives testing for HIV? Journal of Acquired Immunodeficiency Syndrome, 22, 490-497.

Nyamathi, A. M., Stein, J. A. y Swanson, J. M. (2000). Personal, cognitive, behavioral and demographic predictors of HIV testing and STDs in homeless women. Journal of Behavioral Medicine, 23, 123-147.

Piña, J. A. y Bullé, R. (1994). Perspectivas de un modelo psicológico para la prevención y la investigación. Psicología y Salud, 4, 177-185.

Piña, J. A. y Corral, V. (2001). Conocimientos y motivos asociados a comportamientos de riesgo y prevención del SIDA. Avances en Psicología Clínica Latinoamericana, 19, 7-24.

Piña, J. A. y Obregón, F. (en prensa). Algunas reflexiones sobre el concepto de prevención en el contexto de la relación psicología y salud. En J. A. Piña, J. A. Vera y F. J. Obregón (Eds.), Psicología y salud en Iberoamérica. Hermosillo, México: Secretaría de Salud-CIAD-Universidad de Sonora.

Piña, J. A., Duarte, L. y Guzmán, A.C. (1991). El comportamiento como categoría psicológica frente al SIDA. Revista de Psicología Social y Personalidad, 7, 91-99.

Piña, J. A., Márquez, Y. y Vera, J. A. (1992). Información per se versus información como capacidad: implicaciones para la 
prevención del SIDA. En AMEPSO (Eds.), La Psicología Social en México (vol. 4) (pp. 135-140). México, DF: Autor.

Ribes, E. (1990). Psicología y salud: un análisis conceptual. Barcelona: Martínez Roca.

Ribes, E. y López, F. (1985). Teoría de la conducta: un análisis de campo y paramétrico. México, DF: Trillas.

Ribes, E. y Sánchez, S. (1990). El problema de las diferencias individuales: un análisis conceptual de la personalidad. En E. Ribes (Eds.). Problemas conceptuales en el análisis del comportamiento (pp. 79-99). México, DF: Trillas.

Rotheram-Borus, M. J., Gillis, J. R., Reid, M. H., Fernandez, M. J. y Gwadz, M. (1997). HIV testing, behaviors and knowledge among adolescents at high-risk. Journal of Adolescent Health, 20, 216-225.

Silva, A. (1998). La investigación asistida por computadora. México, DF: UNAM.

Wardropper, A. G., Sankar, K. N. y Ong, E. L. (1995). Risk and behavior in patients requesting HIV test. Journal of Infectology, 30, 71-73.

Wenger, N. S., Greenberg, J. M., Hilborne, L. H., Kusseling, F., Mongotich, M. y Shapiro, M. F. (1992). Effect of HIV antibody testing and AIDS education on communication about HIV risk and sexual behavior: A randomized controlled trial in college students. Annals of Internal Medicine, 117, 905-911.

Wolistky, R. J., Mac Gowan, J. R., Higgins, D. L. y Jorgensen, C. M. (1997). The effects of HIV counseling and testing on risk-related practices and help-seeking behavior. AIDS Education and Prevention, 9 (Suppl. 3), 52-67. 